\title{
Urine klotho is a potential early biomarker for acute kidney injury and associated with poor renal outcome after cardiac surgery
}

\author{
Yingying Qian ${ }^{2+}$, Lin Che ${ }^{3+}$, Yucheng Yan ${ }^{1}$, Renhua Lu', Mingli Zhu', Song Xue ${ }^{4}$, Zhaohui Ni ${ }^{1}$ and Leyi Gu*
}

\begin{abstract}
Background: Current paradigms of detecting acute kidney injury (AKI) are insensitive and non-specific. Klotho is a pleiotropic protein that is predominantly expressed in renal tubules. In this study, we evaluated the diagnostic and prognostic roles of urine Klotho for AKI following cardiac surgery.

Methods: We conducted a prospective study involving 91 patients undergoing cardiac surgery. AKI was defined according to the AKIN definition. The renal outcomes within 7 days after operation were evaluated. Perioperative levels of urine Klotho and urine neutrophil gelatinase-associated lipocalin (NGAL) were measured by using ELISA.

Results: Of 91 participants, 33 patients (36.26\%) developed AKI. Of these AKI patients, 21 (63.64\%), 8 (24.24\%), and 4 (12.12\%) were staged 1, 2, and 3, respectively. Serum creatinine in AKI patients began to slightly increase at first postoperative time and reached the AKI diagnostic value 1 day after operation.

Postoperative urine Klotho peaked at the first postoperative time $(0 \mathrm{~h}$ after admission to the intensive care unit $(I C U))$ in patients with AKI, and was higher than that in non-AKI patients up to day 3. The AUC of detecting AKI for urine Klotho was higher than urine NGAL at the first postoperative time and $4 \mathrm{~h}$ after admission to the ICU. In a multivariate model, increased first postoperative urine Klotho may be an independent predictor for AKI occurrence following cardiac surgery. The concentrations of first postoperative urine Klotho were higher in AKI stage 2 and 3 than those in stage $1(p<0.05)$, and were higher in patients with incomplete recovery of renal function than those with complete recovery $(p<0.05)$.
\end{abstract}

Conclusions: Urine Klotho may serve as an early biomarker for AKI and subsequent poor short-term renal outcome in patients undergoing cardiac surgery.

Keywords: Acute kidney injury, Cardiac surgery, Klotho, NGAL, Early biomarker

\section{Background}

Acute kidney injury (AKI) remains a highly prevalent and serious complication of cardiac surgery. AKI develops in up to $40 \%$ of patients who undergo cardiac surgery $[1,2]$. Despite decades of research, the mortality of cardiac surgery-associated AKI (CSA-AKI) remains high, even for those patients whose renal function has completely recovered [3]. A major reason for the disappointing outcome is the scarcity of early biomarkers. Current diagnosis of AKI is made based on the changes

\footnotetext{
*Correspondence: guleyi@aliyun.com

†Yingying Qian and Lin Che contributed equally to this work.

'Department of Nephrology, Renji Hospital, School of Medicine, Shanghai

Jiao Tong University, 160 Pujian Road, Shanghai 200127, China

Full list of author information is available at the end of the article
}

of serum creatinine $(\mathrm{SCr})$ and urine output, which lack sensitivity and reliability [4]. First, SCr reflects the loss of glomerular filtration function rather than the renal tubular lesions. Second, $\mathrm{SCr}$ is vulnerable to several nonrenal factors such as sex, muscle mass, diet and hemodynamic alterations.

Klotho is a multifunctional protein that includes transmembrane and soluble forms [5]. The latter is almost entirely derived from membrane-bound Klotho shedding and has been found in serum, cerebrospinal fluid and urine [6-9]. Soluble Klotho functions as a hormone and plays a role of anti-oxidative stress [10], anti-apoptosis [11], and anti-fibrosis [12]. The renal tubular epithelial cells are the principal cells that contribute to Klotho

(c) The Author(s). 2019 Open Access This article is distributed under the terms of the Creative Commons Attribution 4.0 International License (http://creativecommons.org/licenses/by/4.0/), which permits unrestricted use, distribution, and 
synthesis and excretion [13, 14]. Klotho regulates transporters and ion channels through autocrine or paracrine to the urinary luminal side $[9,14]$. Klotho in the urine can be derived from plasma and interstitium through transcytosis, and can also originate from the tubule through secretion rather than filtered across the glomerular barrier $[9,14]$.

To date, few studies have focused on the urine Klotho in prediction of AKI. In the present study, we firstly assessed the diagnostic and prognostic performance of urine Klotho in patients undergoing cardiac surgery.

\section{Methods}

\section{Patients and samples}

Patients who underwent cardiac surgery at the Cardiology Division of the Renji Hospital, School of Medicine, Shanghai Jiaotong University between 1st October 2012 and 30th June 2013 were enrolled. Patients with chronic kidney disease were excluded [15]. Further exclusion criteria included thyroid disease, preoperative usage of high-dose corticosteroids, pre-existing urinary intact infection, and missing clinical data.

The urine specimens were collected at before surgery and at $0 \mathrm{~h}, 2 \mathrm{~h}, 4 \mathrm{~h}, 1 \mathrm{~d}, 3 \mathrm{~d}$, and $7 \mathrm{~d}$ after admission to the ICU. The blood specimens were collected preoperatively, as well as at $0 \mathrm{~h}, 1 \mathrm{~d}, 2 \mathrm{~d}, 3 \mathrm{~d}$, and $7 \mathrm{~d}$ after arrival to the ICU. The first postoperative samples were collected at $0 \mathrm{~h}$ after arrival to the ICU within $4 \mathrm{~h}$ after surgery. Samples were quickly collected (stayed less than 4 $\mathrm{h}$ at $4{ }^{\circ} \mathrm{C}$ ) and centrifuged at $3000 \mathrm{rpm}$ for $5 \mathrm{~min}$. The supernatants were aliquoted and frozen at $-80^{\circ} \mathrm{C}$.

\section{Variable definitions}

Data including preoperative characteristics, surgical details, and postoperative complications were collected. Diagnosis and staging of postoperative AKI were according to AKIN criteria [16]. AKI was defined as an increase in $\mathrm{SCr}$ of at least $50 \%$ or more than $0.3 \mathrm{mg} / \mathrm{dl}$ from baseline within $48 \mathrm{~h}$. The short-term renal outcome of AKI patients was evaluated according to changes in renal function on day 7 after operation. Complete recovery was defined as reduction of serum creatinine from the peak to less than $0.3 \mathrm{mg} / \mathrm{dl}$ [17]. The eGFR was calculated using Chronic Kidney Disease Epidemiology Collaboration (CKD-EPI) equation [18].

\section{Biomarker assays}

We measured urine Klotho using Klotho ELISA kits (Immuno-Biological Laboratories Co, Tokyo, Japan). We measured urine NGAL using NGAL ELISA kits (R\&D Systems, Inc., Minneapolis, MN, USA). Samples were used with one freeze/thaw cycle and were detected by a technician with a blind method. The intra- and interassay coefficient of variation for Klotho were both $<15 \%$ and for NGAL were both $<10 \%$. The results were corrected for urine creatinine excretion.

\section{Statistical analyses}

Continuous variables were expressed as mean \pm standard deviation, and were compared using $t$ test or one-way ANOVA followed by Tukey multiple comparison tests when they were normally distributed. Non-normally distributed continuous data were expressed as medians with interquartile range, and were analyzed using Wilcoxon rank sum test. Categorical variables were analyzed with Pearson $x^{2}$ test or Fisher's exact test. We plotted the receiver operating characteristic (ROC) curve to measure the sensitivity and specificity of urine Klotho and urine NGAL for AKI prediction at different time cutoffs. The area under the ROC (AUC) was calculated to assess the ability of each biomarker to discriminate between patients developing and those not developing AKI after cardiac surgery. We conducted a univariate analysis for the predictors of AKI. Then, those variables with $p<0.05$ were candidates for multivariate logistic regression models, including operation time and the first postoperative urine Klotho. In the multivariate analysis, we also adjusted for important covariates that predict AKI in the cardiac surgery setting $[19,20]$, including baseline SCr.

SPSS version 19.0 (SPSS Inc., Chicago, Ill., USA) and MedCalc version 18.5 (Ostend, Belgium) for windows software were used for analyses. $P<0.05$ was considered to be statistically significant.

\section{Results}

\section{Patient characteristics}

A total of 103 patients were screened and 91 patients were included in the study. 12 patients were excluded for preexisting urinary intact infection $(n=2)$, pre-existing CKD $(n=3)$, and missing clinical data $(n=7)$. The mean preoperative estimated glomerular filtration rate (eGFR) was $86.89 \pm 15.55 \mathrm{ml} / \mathrm{min} / 1.73 \mathrm{~m}^{2}$. The average age was $61.79 \pm 9.37$ years. 33 patients $(36.26 \%)$ developed AKI at a median time of $24 \mathrm{~h}$ post-surgery. 21 (63.64\%), 8 (24.24\%) and 4 (12.12\%) AKI patients were staged into 1, 2 and 3, respectively. No patient required acute dialysis and 2 died before discharge. The baseline characteristics were showed in Table 1 . No difference between AKI and non-AKI individuals was found in demographics and the types of operation. Patients who developed AKI had longer operation times than those who did not $(5.5(4.5,6.25) \mathrm{h}$ vs. $4.5(4.00,5.50) \mathrm{h}, p<0.05)$.

\section{Perioperative biomarkers concentrations}

There was no difference in preoperative levels of Scr, urine Klotho (uKlotho) and urine NAGL (uNGAL) between 
Table 1 Baseline characteristics of studied population undergoing cardiac surgery

\begin{tabular}{|c|c|c|c|c|}
\hline & All $(n=91)$ & AKI $(n=33)$ & Non-AKI $(n=58)$ & $p$ value \\
\hline \multicolumn{5}{|l|}{ Preoperative condition } \\
\hline Male, $n(\%)$ & $58(63.7 \%)$ & $23(69.7 \%)$ & $35(63.7 \%)$ & 0.372 \\
\hline Age (year) $)^{a}$ & $61.79 \pm 9.37$ & $64.15 \pm 9.37$ & $60.45 \pm 9.18$ & 0.070 \\
\hline Weight $(\mathrm{kg})^{\mathrm{a}}$ & $62.11 \pm 10.29$ & $63.36 \pm 10.06$ & $61.38 \pm 10.45$ & 0.384 \\
\hline Hypertension, $n$ (\%) & $33(36.3 \%)$ & $13(39.4 \%)$ & $20(34.5 \%)$ & 0.639 \\
\hline Diabetes, $n(\%)$ & $14(15.4 \%)$ & $6(18.2 \%)$ & $8(13.8 \%)$ & 0.577 \\
\hline Hyperuricemia, $n$ (\%) & $15(16.5 \%)$ & $6(18.2 \%)$ & $9(15.5 \%)$ & 0.742 \\
\hline Cerebrovascular disease, $n$ (\%) & $4(4.4 \%)$ & $0(0.0 \%)$ & $4(6.9 \%)$ & 0.312 \\
\hline Hyperlipidemia, $n(\%)$ & $6(6.6 \%)$ & $1(3.0 \%)$ & $5(8.6 \%)$ & 0.553 \\
\hline Peripheral vascular disease, $n(\%)$ & $3(3.3 \%)$ & $2(6.1 \%)$ & $1(1.7 \%)$ & 0.615 \\
\hline Congestive heart failure, $n(\%)$ & $13(14.3 \%)$ & $7(21.2 \%)$ & $6(10.3 \%)$ & 0.266 \\
\hline Contrast medium, n (\%) & $14(15.4 \%)$ & $7(21.2 \%)$ & $7(12.1 \%)$ & 0.245 \\
\hline Use of ACE inhibitors/ARBs, $n(\%)$ & $25(27.5 \%)$ & $9(27.3 \%)$ & $16(27.6 \%)$ & 0.974 \\
\hline Hemoglobin $(g / L)^{b}$ & $\begin{array}{l}129.00 \\
(116.00,141.00)\end{array}$ & $\begin{array}{l}124.00 \\
(113.00,141.00)\end{array}$ & $\begin{array}{l}133.00 \\
(120.00,141.50)\end{array}$ & 0.219 \\
\hline Scr $(u m o l / L)^{a}$ & $73.77 \pm 13.88$ & $76.09 \pm 15.60$ & $72.44 \pm 12.76$ & 0.230 \\
\hline eGFR $\left(\mathrm{ml} / \mathrm{min} / 1.73 \mathrm{~m}^{2}\right)^{\mathrm{a}}$ & $86.89 \pm 15.55$ & $88.38 \pm 14.35$ & $84.27 \pm 17.37$ & 0.228 \\
\hline \multicolumn{5}{|l|}{ Intraoperative condition } \\
\hline \multicolumn{5}{|l|}{ Type of surgery } \\
\hline CABG, $n(\%)$ & $32(35.2 \%)$ & $10(30.3 \%)$ & $22(37.9 \%)$ & 0.464 \\
\hline Single valve, $n(\%)$ & $20(22.0 \%)$ & $6(18.2 \%)$ & $14(24.1 \%)$ & 0.509 \\
\hline Double valve, $n(\%)$ & 19 (20.0\%) & $9(27.3 \%)$ & $10(17.2 \%)$ & 0.258 \\
\hline CABG plus valve surgery, $\mathrm{n}(\%)$ & $10(11.0 \%)$ & $4(12.1 \%)$ & $6(10.3 \%)$ & 1.000 \\
\hline CHD, n (\%) & $5(5.5 \%)$ & $2(6.1 \%)$ & $3(5.2 \%)$ & 1.000 \\
\hline Aortic aneurysm surgery, $n$ (\%) & $5(5.5 \%)$ & $2(6.1 \%)$ & $3(5.2 \%)$ & 1.000 \\
\hline $\mathrm{CPB}, n(\%)$ & $67(73.6 \%)$ & $27(81.8 \%)$ & $40(70.2 \%)$ & 0.222 \\
\hline Operation time, $\mathrm{h}^{\mathrm{b*}}$ & $5.00(4.00,6.00)$ & $5.50(4.50,6.25)$ & $4.50(4.00,5.50)$ & 0.002 \\
\hline CPB time $(\mathrm{min})^{\mathrm{b}}$ & $84.00(0.00,115.00)$ & $94.00(56.00,134.00)$ & $76.50(0.00,112.25)$ & 0.223 \\
\hline AXC time $(\min )^{\mathrm{b}}$ & $46.00(0.00,69.00)$ & $55.00(11.50,73.50)$ & $46.00(0.00,66.50)$ & 0.537 \\
\hline Cardiac arrest time $(\mathrm{min})^{\mathrm{b}}$ & $48.00(0.00,74.00)$ & $60.00(13.00,75.00)$ & $46.50(0.00,70.00)$ & 0.526 \\
\hline Transfusion > 400 ml, n (\%) & $10(11.0 \%)$ & $4(12.1 \%)$ & $6(10.3 \%)$ & 1.000 \\
\hline
\end{tabular}

${ }^{\mathrm{a} D a t a}$ were presented as means \pm standard deviation (SD) and the difference were calculated using $t$ test. ${ }^{\mathrm{b}}$ Data were presented as medians (IOR, interquartile range) and were analyzed using non-parametric Mann-Whitney $U$ test. Categorical variables were presented as number (percentage of the column total) and were analyzed using Pearson $x 2$ test. ${ }^{*} p<0.05$

$A C E$ Angiotensin-converting enzyme, ARB Angiotensin receptor blockers, $S C r$ Serum creatinine, eGFR Estimated glomerular filtration rate, CABG Coronary artery bypass grafting, CHD Congenital heart disease, CPB Cardiopulmonary bypass, AXC Aortic cross clamp

patients with and without AKI. Serum creatinine in AKI patients began to slightly increase at first postoperative time $(88.00(78.35,111.30)$ umol/L vs. preoperative: 76.80 $(64.20,90.45) \mathrm{umol} / \mathrm{L}, p<0.01)$ and reached the AKI diagnostic value 1 day after arrival to the ICU (Fig. 1a). The levels of urine Klotho peaked at first postoperative time in patients with AKI and were 3 times higher than that before surgery $(1.69(1.02,2.68) \mathrm{ng} / \mathrm{umol}$ vs. $0.41(0.30,0.65)$ $\mathrm{ng} / \mathrm{umol}, p<0.001$, Fig. 1b). Further, urine Klotho levels in AKI patients were strikingly higher than those in non-AKI patients at the first postoperative time $(1.69(1.02,2.68)$ ng/umol vs. $0.52(0.23,0.84) \mathrm{ng} / \mathrm{umol}, p<0.01)$, and remained significantly higher up to day 3 postoperatively. The levels of urine NGAL in patients who developed AKI peaked at $2 \mathrm{~h}$ after admission to the ICU, but recovered at $4 \mathrm{~h}$. Urine NGAL in patients who developed AKI was significantly higher than those who did not at $2 \mathrm{~h}$ (Fig. 1c).

\section{AUC analyses for cardiac surgery-associated AKI}

We evaluated the AUCs for biomarkers in predicting AKI at indicated time points. As shown in Table 2, the AUC for uKlotho at the first postoperative time was 0.86 




with the cutoff $0.86 \mathrm{ng} /$ umol (sensitivity $93.9 \%$, specificity 75.9\%). Urine Klotho had higher AUCs than uNGAL at the first postoperative time and $4 \mathrm{~h}$ after arrival to the ICU $(p<0.001)$.

\section{The first postoperative urine klotho was associated with} the occurrence of AKI

As shown in Table 3, the first postoperative uKlotho was associated with AKI occurrence with unadjusted and adjusted odds ratio (OR) 3.36 (95\% CI, 1.86 to
6.07) and 3.43 (95\% CI, 1.87 to 6.28), respectively. The data also showed that the risk of AKI occurrence increased by 1.5 times for each additional hour of operation.

\section{Perioperative levels of uKlotho among different stages of AKI}

Compared with non-AKI patients, patients with AKI stage 1 had significantly higher levels of uKlotho at the first postoperative time (Fig. 2). Patients who experienced AKI stage

Table 2 AUC of biomarkers to predict AKI after cardiac surgery

\begin{tabular}{|c|c|c|c|}
\hline & AUC (95\% Cl) & Cut-off (Sensitivity, Specificity) & $p$ value \\
\hline \multicolumn{4}{|l|}{ Urine Klotho (ng/umol) } \\
\hline The first postoperative & $0.86^{* * *}(0.78,0.94)$ & $0.86(93.9,75.9 \%)$ & $<0.001$ \\
\hline $2 \mathrm{~h}$ admission to the ICU & $0.85(0.76,0.94)$ & $0.82(87.5,77.6 \%)$ & $<0.001$ \\
\hline $4 \mathrm{~h}$ admission to the ICU & $0.78^{* * *}(0.76,0.94)$ & $0.70(90.6,62.1 \%)$ & $<0.001$ \\
\hline \multicolumn{4}{|l|}{ Urine NGAL (ng/umol) } \\
\hline The first postoperative & $0.59(0.46,0.71)$ & $4.39(72.7,56.6 \%)$ & 0.177 \\
\hline $2 \mathrm{~h}$ admission to the ICU & $0.78(0.68,0.88)$ & $4.49(87.9,71.7 \%)$ & $<0.001$ \\
\hline $4 \mathrm{~h}$ admission to the ICU & $0.57(0.44,0.70)$ & $4.64(57.6,64.2 \%)$ & 0.285 \\
\hline
\end{tabular}

AUC Area under the ROC, NGAL Neutrophil gelatinase-associated lipocalin

${ }^{* * *} P<0.001$ compared with the AUCs of urine NGAL at indicated time points 
Table 3 Association of first postoperative urine Klotho with AKI



2 and 3 had higher levels of first postoperative uKlotho than patients with stage $1(2.68(1.54,5.06) \mathrm{ng} / \mathrm{umol}$ vs. $1.30(0.97,2.27) \mathrm{ng} / \mathrm{umol}, P<0.05)$. Levels of uKlotho in patients with AKI stage 2 and 3 were markedly higher than that in non-AKI patients at the first postoperative time and remained higher up to day $7(P<0.01)$.

\section{Perioperative levels of uKlotho among non-AKI, AKI with} complete recovery, and AKI with incomplete recovery As shown in Fig. 3, 21 (63.63\%) AKI patients had complete recovery of renal function on day 7 . The levels of first postoperative uKlotho were $0.52(0.23,0.84)$ ng/umol, 1.3 (0.97, 2.34) ng/umol, and $2.58(1.41,3.60) \mathrm{ng} / \mathrm{umol}$ in patients with non-AKI, complete recovery AKI, and incomplete recovery AKI, respectively $(p<0.01)$.

\section{Discussion}

To our knowledge, we are the first to examine the time course of uKlotho among adults undergoing cardiac surgery. We found that the elevated first postoperative
uKlotho may be an early predicator for the occurrence of CSA-AKI. Further, urine Klotho in patients with AKI stage 2 and 3 was higher than that in patients with AKI stage 1 at the first postoperative time. Urine Klotho in AKI patients with incomplete recovery of renal function was significantly higher than that in patients with complete recovery at the first postoperative time.

AKI following cardiac surgery is a world health issue. Serum creatinine and urine output remains the gold standard for clinical diagnosis of AKI, although both were believed as unspecific markers for kidney injury. Novel biomarkers including NGAL, KIM-1 and interleukin-18 (IL-18) were evaluated for early diagnosis of AKI. Unlike Klotho that mainly expressed in kidney tubule, NGAL and IL-18 are nonspecific for kidney and are expressed in variety of tissues [4, 21]. Moreover, large, prospective, multicenter trails failed to show troponin-like diagnostic performance of plasma NGAL, urine NGAL, urine Kim-1, and urine IL-18 for AKI detecting with AUCs of less than 0.77 [22, 23]. Thus, efforts to validate potential markers are needed.

In animal experiments, Klotho deficiency in kidney tissues has been observed in both acute and chronic kidney injuries $[24,25]$. More recently, a small study with 35 patients observed reduced serum Klotho in adults who developed AKI after cardiac surgery [26]. However, no significant difference of serum Klotho was found between patients with and without AKI on 1 day postoperatively and thereafter. Moreover, their study did not evaluate the change of urine Klotho and did not comment on the severity or renal outcome of AKI.

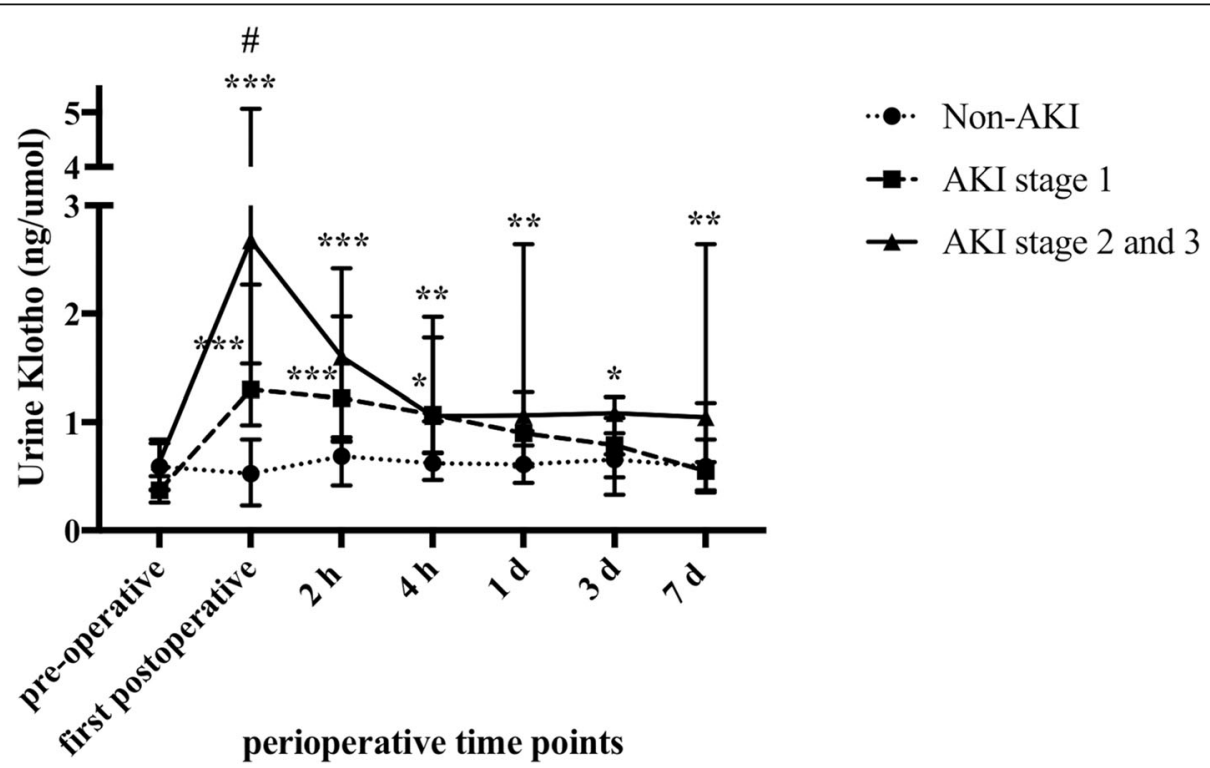

Fig. 2 Perioperative levels of uKlotho among different stages of AKI. Patients were grouped as non-AKI, AKI stage 1, and AKI stage 2 and 3. The levels of uKlotho were analyzed. Urine Klotho was corrected for urine creatinine excretion. Data were presented as median with interquartile range. ${ }^{\#}$-value $<0.05$ vs. AKI stage 1 group. ${ }^{*} P$-value $<0.05$, ${ }^{* *} p$-value $<0.01$, and ${ }^{* * *} p$-value $<0.001$ vs. non-AKI patients 




Fig. 3 Perioperative levels of uKlotho among non-AKI, AKI with complete recovery, and AKI with incomplete recovery. Levels of uKlotho among 3 groups were analyzed. Data were presented as median with interquartile range. ${ }^{\# P}$-value $<0.05$ vs. AKI with complete recovery. ${ }^{*} P$-value $<0.05$, ${ }^{* *} p$-value $<0.01$, and ${ }^{* * *} p$-value $<0.001$ vs. non-AKI patients

We firstly showed that uKlotho quickly increased as early as transferred to the ICU in patients who developed AKI later. Urine Klotho kept significantly higher in AKI patients than non-AKI patients until day 3 after surgery. The elevation of uKlotho occurred earlier than that of UNGAL demonstrated in the present cohorts and our previous data [27]. However, there was no difference in preoperative levels of uKlotho between patients with and without AKI. The early performance of urine Klotho may allow earlier detection of AKI and thus increase the success of therapeutic interventions.

To date, only two studies had examined uKlotho levels in patients or rodents with AKI. Isidro and his colleagues [28] examined the levels of uKlotho at $12 \mathrm{~h}$ post-cardiac surgery and showed a similar elevation of that in AKI patients $(n=15)$ compared with the healthy volunteers $(n=10)$. When comparing to the non-AKI $(n=15)$ group, the uKlotho levels in AKI patients increased but without significance. However, our results conflict with the reports from $\mathrm{Hu}$ et al. [24]. They found that urine Klotho levels in 17 AKI patients were significantly lower than that in 14 healthy controls by using immunoblotting assay. There are several reasons for the conflicted results. First, $\mathrm{Hu}$ did not describe the methods of pretreatment and the collecting time of urine samples. Klotho is unstable in urine and any additional freeze-thaw cycle decreases Klotho concentrations [29]. In the present study, urine samples were frozen at $-80^{\circ} \mathrm{C}$ and were thawed for the first time for measurement of Klotho. Furthermore, uKlotho in patients with AKI was comparable with that in patients without AKI on 7 days post-surgery. Thus, a delayed collecting time may lead to different results. Second, the different causes of AKI may also lead to the contrary results. In the study of $\mathrm{Hu}$ et al., the causes of AKI are heterogeneous including sepsis, hypertension, CKD, nephrontoxin, pre-renal and others. Different measuring assays and fewer patients may also contribute the difference. Further researches with large sample size, multi-center and extensive time course are needed to clarify the change pattern of uKlotho.

Histologically, proximal tubular epithelial cells lose their brush border membrane as well as the Klotho protein expressed in the apical brush border, which may result in an acute increase of uKlotho at early AKI. With the progression of AKI, renal tubular epithelial cells were necrotic and exfoliated, accompanied with shedding of Klotho protein. This may contribute to the continuous elevation of uKlotho during AKI. We have previously showed similar phenomenon in the mouse model of AKI induced by renal ischemic-reperfusion injury [30]. Under these circumstances, the increase of uKlotho may indicate the severity of tubule injury. In support of this, we found that uKlotho was strongly associated with the occurrence of CSA-AKI and was significantly higher in AKI stage 2 and 3 than in stage 1. 
Our study has several limitations. First, The present study is a single center study with a relatively small number of patients and short follow-up. Second, we did not compare the performance of predicting AKI between Klotho and other biomarkers besides NGAL. Third, the time points of uNGAL detection are insufficient. Additionally, the use of creatinine as a reference standard for biomarker assessment is imperfect for the known insensitivity and non-specificity.

\section{Conclusions}

In conclusion, our study implies that first postoperative uKlotho may be an early predictor for the occurrence of AKI among patients undergoing cardiac surgery. The first postoperative uKlotho may be associated with the severity and prognosis of AKI. This may help to early identify at-risk patients before the progression to overt kidney injury and implement early interventions.



Abbreviations

ACE: Angiotensin-converting enzyme; AKI: Acute kidney injury; AKIN: Acute Kidney Injury Network; ARB: Angiotensin receptor blockers; AUC: Area under the ROC; AXC: Aortic cross clamp; CABG: Coronary artery bypass grafting; CHD: Congenital heart disease; CKD: Chronic kidney disease; CKDEPI: Chronic Kidney Disease Epidemiology Collaboration; CPB: Cardiopulmonary bypass; CSA-AKI: Cardiac surgery-associated acute kidney injury; eGFR: Estimated glomerular filtration rate;" ICU: Intensive care unit; KDOQI: Kidney Disease Outcome Quality Initiative; Kim-1: Kidney injury molecule-1; NGAL: Neutrophil gelatinase-associated lipocalin; OR: Odds rate; ROC: Receiver operating characteristic; SCr: Serum creatinine; UUO: Unilateral ureteral obstruction

\section{Acknowledgements}

Not applicable.

\section{Authors' contributions}

YQ analyzed and interpreted the patient data, and drafted the manuscript. LC performed data collection and biomarkers examination. YY conceived and designed the experiments and contributed reagents and materials. RL, $M Z$, and SX participated in data collection. ZN contributed reagents and materials. LG critically revised the manuscript for important intellectual content, and contributed reagents and materials. All authors read and approved the final manuscript.

\section{Funding}

The design of the study and ELISA kits were supported by the National Natural Science Foundation of China (No. 81170678, NO.81470918). Experimental consumables, data collection and analysis were supported by Natural Science Foundation of Shanghai (No.15ZR1425900) and Shanghai science and technology commission (No.17695840500).

\section{Availability of data and materials}

The datasets used and/or analyzed during this study are available from the corresponding author on reasonable request.

\section{Ethics approval and consent to participate}

This study was approved by the ethical review board of Renji Hospital, School of Medicine, Shanghai Jiaotong University. All of the patients were given and accepted written informed consent prior to enrollment.

\section{Consent for publication}

Not applicable.

\section{Competing interests}

The authors declare that they have no competing interests.

\section{Author details}

'Department of Nephrology, Renji Hospital, School of Medicine, Shanghai Jiao Tong University, 160 Pujian Road, Shanghai 200127, China. ²Department of Nephrology, Affiliated Hangzhou First People's Hospital, Zhejiang University School of Medicine, Hangzhou, China. ${ }^{3}$ Department of Nephrology, The Affiliated Hospital of Qingdao University, Qingdao, China. ${ }^{4}$ Department of Cardiovascular Surgery, Renji Hospital, School of Medicine, Shanghai Jiao Tong University, Shanghai, China.

Received: 29 November 2017 Accepted: 9 July 2019

Published online: 17 July 2019

\section{References}

1. Tuttle KR, Worrall NK, Dahlstrom LR, Nandagopal R, Kausz AT, Davis CL. Predictors of ARF after cardiac surgical procedures. Am J Kidney Dis. 2003; 41(1):76-83

2. Rosner MH, Okusa MD. Acute kidney injury associated with cardiac surgery. Clin J Am Soc Nephrol. 2006;1(1):19-32.

3. Hobson CE, Yavas S, Segal MS, Schold JD, Tribble CG, Layon AJ, et al. Acute kidney injury is associated with increased long-term mortality after cardiothoracic surgery. Circulation. 2009;119(18):2444-53.

4. Makris K, Kafkas N. Neutrophil gelatinase-associated lipocalin in acute kidney injury. Adv Clin Chem. 2012;58:141-91.

5. Xu Y, Sun Z. Molecular basis of klotho: from gene to function in aging. Endocr Rev. 2015;36(2):174-93.

6. Mencke R, Harms G, Moser J, van Meurs M, Diepstra A, Leuvenink HG, et al. Human alternative Klotho mRNA is a nonsense-mediated mRNA decay target inefficiently spliced in renal disease. JCI Insight. 2017;2(20).

7. Imura A, Iwano A, Tohyama O, Tsuji Y, Nozaki K, Hashimoto N, et al. Secreted klotho protein in sera and CSF: implication for post-translational cleavage in release of klotho protein from cell membrane. FEBS Lett. 2004; 565(1-3):143-7.

8. Barker SL, Pastor J, Carranza D, Quinones H, Griffith C, Goetz R, et al. The demonstration of alphaKlotho deficiency in human chronic kidney disease with a novel synthetic antibody. Nephrol Dial Transplant. 2015;30(2):223-33.

9. Hu MC, Shi M, Zhang J, Pastor J, Nakatani T, Lanske B, et al. Klotho: a novel phosphaturic substance acting as an autocrine enzyme in the renal proximal tubule. FASEB J. 2010;24(9):3438-50.

10. Wang Y, Kuro-o M, Sun Z. Klotho gene delivery suppresses Nox2 expression and attenuates oxidative stress in rat aortic smooth muscle cells via the CAMP-PKA pathway. Aging Cell. 2012;11(3):410-7.

11. Lin $Y$, Sun Z. Antiaging gene klotho attenuates pancreatic beta-cell apoptosis in type 1 diabetes. Diabetes. 2015;64(12):4298-311.

12. Hu MC, Shi M, Gillings N, Flores B, Takahashi M, Kuro OM, et al. Recombinant alpha-klotho may be prophylactic and therapeutic for acute to chronic kidney disease progression and uremic cardiomyopathy. Kidney Int. 2017;91(5):1104-14.

13. Lindberg K, Amin R, Moe OW, Hu MC, Erben RG, Ostman Wernerson A, et al The kidney is the principal organ mediating klotho effects. J Am Soc Nephrol. 2014;25(10):2169-75.

14. Hu MC, Shi M, Zhang J, Addo T, Cho HJ, Barker SL, et al. Renal production, uptake, and handling of circulating alphaKlotho. J Am Soc Nephrol. 2016; 27(1):79-90.

15. National KF. K/DOQI clinical practice guidelines for chronic kidney disease: evaluation, classification, and stratification. Am J Kidney Dis. 2002;39(2 Suppl 1):S1-266.

16. Mehta RL, Kellum JA, Shah SV, Molitoris BA, Ronco C, Warnock DG, et al. Acute kidney injury network: report of an initiative to improve outcomes in acute kidney injury. Crit Care. 2007;11(2):R31.

17. Xie Y, Wang Q, Wang C, Qi C, Ni Z, Mou S. High urinary excretion of kidney injury molecule-1 predicts adverse outcomes in acute kidney injury: a case control study. Crit Care. 2016;20:286.

18. Levey AS, Stevens LA, Schmid CH, Zhang YL, Castro AF 3rd, Feldman HI, et al. A new equation to estimate glomerular filtration rate. Ann Intern Med. 2009;150(9):604-12.

19. Che M, Li Y, Liang X, Xie B, Xue S, Qian J, et al. Prevalence of acute kidney injury following cardiac surgery and related risk factors in Chinese patients. Nephron Clin Pract. 2011;117(4):C305-11.

20. Thakar CV, Arrigain S, Worley S, Yared JP, Paganini EP. A clinical score to predict acute renal failure after cardiac surgery. J Am Soc Nephrol. 2005; 16(1):162-8. 
21. Siew ED, Ware LB, Ikizler TA. Biological markers of acute kidney injury. J Am Soc Nephrol. 2011;22(5):810-20.

22. Parikh CR, Coca SG, Thiessen-Philbrook H, Shlipak MG, Koyner JL, Wang Z, et al. Postoperative biomarkers predict acute kidney injury and poor outcomes after adult cardiac surgery. J Am Soc Nephrol. 2011;22(9):1748-57.

23. Parikh CR, Thiessen-Philbrook H, Garg AX, Kadiyala D, Shlipak MG, Koyner JL, et al. Performance of kidney injury molecule-1 and liver fatty acid-binding protein and combined biomarkers of AKI after cardiac surgery. Clin J Am Soc Nephrol. 2013;8(7):1079-88.

24. Hu MC, Shi M, Zhang J, Quinones H, Kuro-o M, Moe OW. Klotho deficiency is an early biomarker of renal ischemia-reperfusion injury and its replacement is protective. Kidney Int. 2010;78(12):1240-51.

25. Shimamura Y, Hamada K, Inoue K, Ogata K, Ishihara M, Kagawa T, et al. Serum levels of soluble secreted alpha-klotho are decreased in the early stages of chronic kidney disease, making it a probable novel biomarker for early diagnosis. Clin Exp Nephrol. 2012;16(5):722-9.

26. Liu YJ, Sun HD, Chen J, Chen MY, Ouyang B, Guan XD. Klotho: a novel and early biomarker of acute kidney injury after cardiac valve replacement surgery in adults. Int J Clin Exp Med. 2015;8(5):7351-8.

27. Che M, Xie B, Xue S, Dai H, Qian J, Ni Z, et al. Clinical usefulness of novel biomarkers for the detection of acute kidney injury following elective cardiac surgery. Nephron Clin Pract. 2010;115(1):c66-72.

28. Torregrosa I, Montoliu C, Urios A, Gimenez-Garzo C, Tomas P, Solis MA, et al. Urinary klotho measured by ELISA as an early biomarker of acute kidney injury in patients after cardiac surgery or coronary angiography. Nefrologia. 2015:35(2):172-8

29. Adema AY, Vervloet MG, Blankenstein MA, Heijboer AC. Alpha-klotho is unstable in human urine. Kidney Int. 2015;88(6):1442-4

30. Qian Y, Guo X, Che L, Guan X, Wu B, Lu R, et al. Klotho reduces necroptosis by targeting oxidative stress involved in renal ischemic-reperfusion injury. Cell Physiol Biochem. 2018;45(6):2268-82.

\section{Publisher's Note}

Springer Nature remains neutral with regard to jurisdictional claims in published maps and institutional affiliations.

Ready to submit your research? Choose BMC and benefit from:

- fast, convenient online submission

- thorough peer review by experienced researchers in your field

- rapid publication on acceptance

- support for research data, including large and complex data types

- gold Open Access which fosters wider collaboration and increased citations

- maximum visibility for your research: over $100 \mathrm{M}$ website views per year

At $\mathrm{BMC}$, research is always in progress.

Learn more biomedcentral.com/submissions 